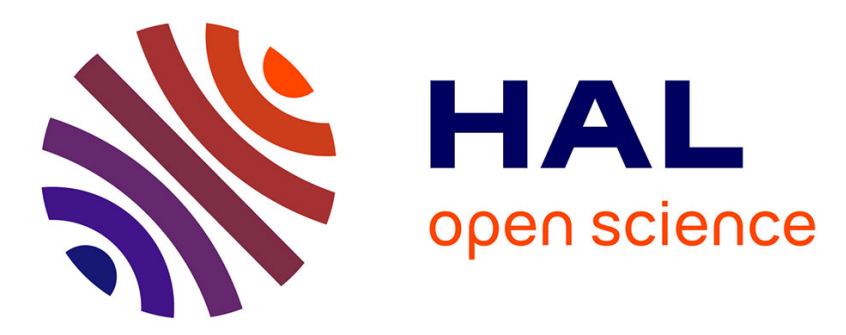

\title{
Dispositif expérimental d'analyse spectrale pour l'étude de la cathodoluminescence; Application au Zn Se
}

\author{
J.M. Guillard, G. Hitier
}

\section{To cite this version:}

J.M. Guillard, G. Hitier. Dispositif expérimental d'analyse spectrale pour l'étude de la cathodoluminescence; Application au Zn Se. Revue de Physique Appliquée, 1973, 8 (4), pp.337-340. 10.1051/rphysap:0197300804033700 . jpa-00243692

\section{HAL Id: jpa-00243692 https://hal.science/jpa-00243692}

Submitted on 1 Jan 1973

HAL is a multi-disciplinary open access archive for the deposit and dissemination of scientific research documents, whether they are published or not. The documents may come from teaching and research institutions in France or abroad, or from public or private research centers.
L'archive ouverte pluridisciplinaire HAL, est destinée au dépôt et à la diffusion de documents scientifiques de niveau recherche, publiés ou non, émanant des établissements d'enseignement et de recherche français ou étrangers, des laboratoires publics ou privés. 


\title{
DISPOSITIF EXPÉRIMENTAL D'ANALYSE SPECTRALE POUR L'ÉTUDE DE LA CATHODOLUMINESCENCE ; APPLICATION AU ZnSe
}

\author{
J. M. GUILlaRD $\left({ }^{*}\right)$ et G. HITIER $\left({ }^{* *}\right)$
}

Laboratoire de luminescence 1, 9 quai Saint-Bernard, 75-Paris $5^{\mathrm{e}}$, France

(Reçu le 11 octobre 1972)

\begin{abstract}
Résumé. - Nous décrivons ici un ensemble électronique utilisé pour des expériences de cathodoluminescence en régime d'impulsions. Il assure l'enregistrement automatique du spectre et comporte des programmes permettant de faire varier le débit, la densité, la durée de bombardement et le temps de repos entre chaque groupe d'impulsions. Cet appareil a été testé sur différents cristaux de $\mathrm{ZnSe}$ cubique.
\end{abstract}

Abstract. - We describe here an electronic apparatus for the automatic recording and display of spectra in pulsed cathodoluminescence experiments. In our apparatus the pulse current, the repetition rate of the pulses, the duration of each group of pulses, and the rest time between groups of pulses may all be programmed. This apparatus has been tested by performing measurements on several different cubic $\mathrm{ZnSe}$ crystals.

La cathodoluminescence a contribué depuis quelques années à améliorer notre connaissance des structures de bandes et des niveaux d'impuretés dans les cristaux semi-conducteurs [1] à [6]. C'est pourquoi, nous nous sommes attachés à l'étude des transitions lumineuses émises au voisinage de la bande d'absorption par le $\mathrm{ZnSe}$ lorsqu'il est soumis à une excitation cathodique. A cette fin nous avons été amenés à mettre au point un dispositif expérimental permettant la mesure et le contrôle précis des conditions d'excitation des cristaux et de la répartition de l'intensité $I$ émise par les différents points du cristal. Les principales difficultés rencontrées, les moyens mis en œuvre pour y remédier, ainsi que les premiers résultats expérimentaux seront décrits dans cette publication.

Rappelons tout d'abord que l'étude de la luminescence des cristaux ou poudres soumis à un bombardement électronique (cathodoluminescence) présente de nombreux avantages par rapport aux excitations ultraviolette ou infrarouge : densité de bombardement élevée, profondeur de pénétration des électrons primaires variable à volonté, contrôle des paramètres d'excitation, etc... Cependant, on sait que la répartition spectrale et l'intensité des raies émises par les échantillons dépendent considérablement des paramètres d'excitation et de la température. La tension d'accélé-

(*) 11, avenue de Villars, 78150 Le Chesnay.

(**) Laboratoire de Luminescence, 9, quai Saint-Bernard, 75005 Paris $5^{\mathrm{e}}$. ration des électrons primaires (variable entre 0 et $30 \mathrm{kV}$ ), intervient sur la profondeur de pénétration de ces électrons qui excitent soit des zones de surface, pour des faibles tensions, soit des zones à l'intérieur du cristal pour des tensions élevées (supérieures à $15 \mathrm{kV}$ ). Les autres paramètres comme le débit, la densité, et la durée plus ou moins importante de bombardement provoquent un échauffement de l'échantillon; la largeur de la bande interdite étant étroitement liée à la température, on observe un déplacement des longueurs d'ondes de certaines transitions lumineuses émises par le cristal lorsque la température varie. C'est ce qui se produit lors de l'analyse spectrale dans un intervalle de longueurs d'onde donné. Les premières raies auront été observées alors que le cristal aura reçu une certaine énergie qui le porte à une température $t$; le bombardement continu augmente son échauffement et les raies suivantes seront observées sur un cristal ne se trouvant plus dans les mêmes conditions de températures d'où résulte une erreur quant à la position et à l'intensité des raies d'un même spectre.

Pour remédier à ces inconvénients il faut que les paramètres électriques d'excitation soient parfaitement définis et que la température de l'échantillon reste constante pendant la durée d'enregistrement d'un spectre. Cela a été obtenu grâce à un dispositif n'autorisant la mesure que lorsque les fluctuations (inévitables) des conditions d'excitation restent inférieures à des limites préétablies. En ce qui concerne la température, nous avons pensé limiter son influence sur les 
répartitions spectrales en modulant le faisceau électronique excitateur. Pour cela, nous avons, à chaque longueur d'onde de détection, excité le cristal par un train d'impulsions composé de signaux rectangulaires de largeur égale au 1/100 du temps séparant chaque impulsion. De plus, nous avons choisi une fréquence de travail de telle sorte que pendant le déplacement du réseau pour le choix d'une autre longueur d'onde (période pendant laquelle nous ne bombardons pas le cristal) la longueur du train d'impulsions soit égale à $1 / 100 \mathrm{du}$ temps nécessaire au choix d'une autre longueur d'onde.

$\mathrm{Si}$, malgré les précautions ainsi prises, il s'avère que l'équilibre thermique de l'échantillon est troublé d'une façon notable par l'irradiation, nous pourrons évidemment modifier en conséquence les paramètres d'excitation. Les fluctuations inévitables des faibles flux lumineux émis et les bruits des amplificateurs ont été minimisés pour chaque longueur d'onde par des mesures statistiques des amplitudes.

1. Dispositif expérimental. - Le montage expérimental représenté schématiquement figure 1 comprend :

a) Un canon à électrons de type triode ayant l'anode à la masse et la cathode au - HT. La haute tension, délivrée par un générateur «Sorensen » type 1061, peut varier de 0 à $60 \mathrm{kV}$. Le débit du tube

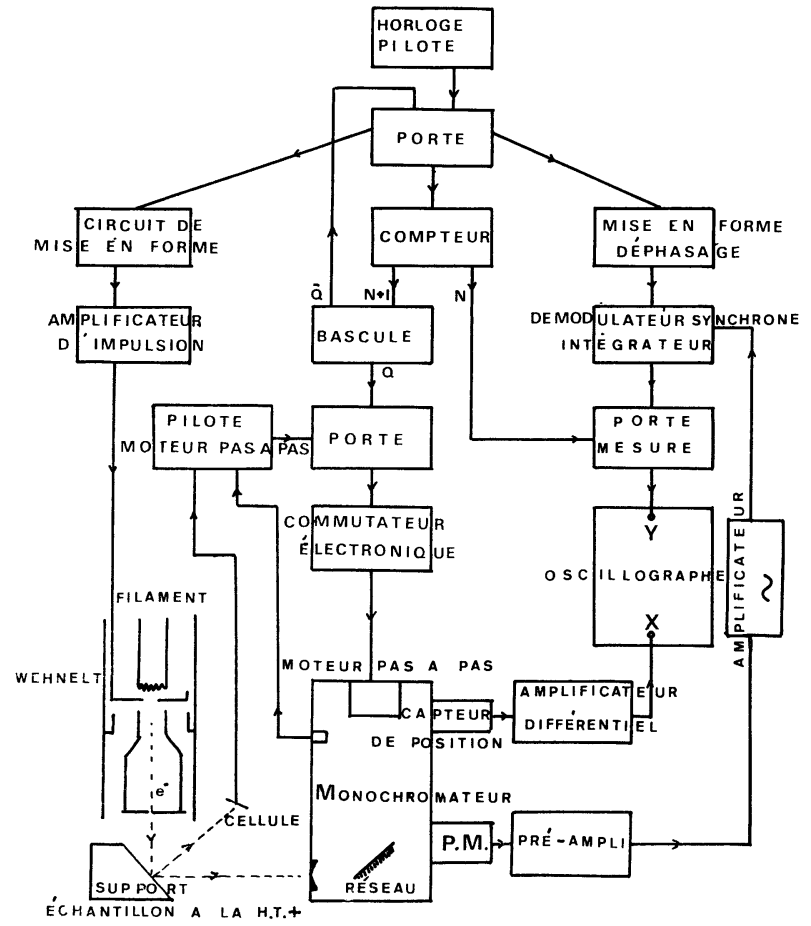

FIG. 1. - Schéma synoptique du montage expérimental.

peut atteindre une valeur maximale de $1 \mathrm{~mA}$. Les alimentations des bobines de déviation et de concentration ainsi que l'alimentation du filament, toutes régulées, à $10^{-4}$ ont été décrites par ailleurs [7]. b) Un cryostat en verre à double paroi qui permet de descendre à une température voisine de celle de l'hélium liquide. Les échantillons sont collés à l'aide d'une peinture à l'argent sur une pièce en argent en contact avec le liquide réfrigérant $\left(\mathrm{He}\right.$ ou $\left.\mathrm{N}_{2}\right)$ de façon à assurer un meilleur écoulement thermique à la température de $\mathrm{He}$ liquide.

c) Un monochromateur à réseau de la société «Physique et Industrie » de type Ebert Fastie, dont le mouvement de rotation est assuré par l'intermédiaire d'un moteur pas à pas.

d) Un ensemble électronique assurant le contrôle, la programmation et les mesures de l'expérience.

L'ensemble électronique est commandé par une horloge pilote qui délivre des impulsions de fréquence variable entre 10 et $3000 \mathrm{~Hz}$. Un circuit de mise en forme fait varier la largeur de chacune des impulsions entre $0,5 \mu$ s et $10 \mathrm{~ms}$, leur tension étant de $5 \mathrm{~V}$. Elles sont alors amplifiées puis dirigées sur l'électrode de Wehnelt du canon à électrons. Ayant une amplitude réglable de $-30 \mathrm{~V}$ à $0 \mathrm{~V}$, une fréquence et une largeur ajustable, elles permettent d'assurer un bombardement par impulsions d'intensité variable, et de laisser au cristal un temps de repos suffisant pour retourner à son équilibre thermique.

Un compteur dont l'entrée est reliée à l'horloge, déclenche la mesure à la $N$-ième impulsion, et par l'intermédiaire d'une bascule arrête mesure et bombardement à la $N+I$-ième impulsion. Simultanément les impulsions destinées à l'avancement du moteur pas à pas sont envoyées sur un commutateur électronique commandant la mise en marche du moteur pas à pas et par conséquent la rotation du réseau. Ces dernières sont produites par un multivibrateur de technologie identique à celle de l'horloge mais travaillant à une fréquence plus basse, compatible avec les caractéristiques du moteur (240 pas/s, angle de rotation/pas de $7^{\circ} 30^{\prime}$ et couple nominal de maintien de $950 \mathrm{~cm} / \mathrm{g}$ ). Un circuit monostable permet par l'intermédiaire de capacités et résistances extérieures de faire varier la durée d'avancement du réseau et par suite l'intervalle en longueur d'onde entre chaque point de mesure (de $0,5 \AA$ à $10 \AA$ ). On réalise ainsi une séquence de manipulation qui comporte le bombardement, la mesure et le changement de longueur d'onde.

Pendant la période de bombardement, la lumière émise par l'échantillon est analysée par le monochromateur puis reçue sur un photomultiplicateur (PM) 56 AVP de la Radiotechnique. Le signal électrique apparaissant sur la résistance de charge du PM est l'image électrique du signal optique délivré par le cristal à une longueur d'onde donnée. Il se présente comme une suite d'impulsions de largeur identique et d'amplitude théoriquement constante à l'intérieur de chaque séquence. Un amplificateur à faible bruit $(20 \mathrm{nV} / \sqrt{\mathrm{Hz}})$, d'un gain de 240000 ramené à 100 par contre-réaction les amène à une valeur suffisante pour être reçues sur l'appareil de lecture. Un commutateur 
synchrone bloque la sortie de l'amplificateur en l'absence de signal et la débloque en sa présence. La commande en phase du commutateur est obtenue grâce à la fréquence pilote de l'horloge mise en forme et convenablement retardée par un double circuit monostable. Un intégrateur branché en sortie permet alors de moyenner la mesure sur les $N+I$ impulsions.

La visualisation s'effectue sur un oscilloscope dont l'entrée $\mathrm{Y}$ est reliée à la chaîne de mesure du signal lumineux par l'intermédiaire d'une porte dont le temps et la durée d'ouverture sont définis respectivement par les nombres $N$ et $I$ avec $N>I$ et variant de 10 en 10 jusqu'à 100.

L'entrée $\mathrm{X}$ commandant la déviation horizontale du spot de l'oscillographe est asservie au mouvement du réseau par le jeu d'un capteur potentiométrique de déplacement de résolution «infinie ». Le signal électrique obtenu est comparé dans un amplificateur différentiel fortement contre-réactionné, à une tension obtenue dans des conditions similaires ce qui rend la dérive des mesures négligeable. Le réseau avançant par à-coups, et la mesure s'effectuant lorsque le réseau est arrêté, on obtient, sur l'oscilloscope et pour chaque longueur d'onde, un point représentant l'intensité émise par le cristal à cette longueur d'onde d'où l'allure du spectre lumineux produit par l'échantillon.

Le contrôle des paramètres d'excitation s'effectue au moyen d'une cellule photoélectrique qui délivre un signal proportionnel à la lumière globale émise par le cristal et traduit donc les fluctuations de celle-ci proportionnellement aux paramètres d'excitation. Un détecteur de seuil impose des limites à ces fluctuations en bloquant le générateur des impulsions du moteur pas à pas, et, le déroulement de la manipulation s'arrête dès qu'il s'est produit soit un déplacement du spot dû aux charges d'espaces qui peuvent s'accumuler sur le cristal soit une fluctuation des conditions d'excitation. Il ne se produit alors d'erreur que sur un seul point de mesure et l'expérimentateur en est averti.

La fin de la manipulation est obtenue par un minirupteur qui bloque l'avancement du réseau.

2. Résultats expérimentaux. - Nous avons sélectionné un certain nombre d'échantillons de cristaux de $\mathrm{ZnSe}$ qui nous ont été offerts soit par « l'Aeronautical Research Laboratory» soit par «Matsushita Electric Industrial Co Ltd ».

Nous avons pu dans une première analyse mettre en évidence 2 types de cristaux comme l'a fait Reynolds [8]. En effet sous l'action d'un bombardement électronique avec des électrons accélérés par une ddp de $15 \mathrm{kV}$ (débit moyen $0,1 \mathrm{~A} / \mathrm{cm}^{2}$ ) et des largeurs d'impulsions de $100 \mu$ s à une fréquence de $1 \mathrm{kHz}$ et pour une température voisine de $13 \mathrm{~K}$, nous avons obtenu, au voisinage de la bande d'absorption 2 types de répartition spectrale de la lumière émise par le $\mathrm{ZnSe}$.

Les cristaux de type I de couleur violette sous excitation cathodique montrent (Fig. 2), du côté des grandes énergies supérieures à $2,8 \mathrm{eV}$, une faible bande $E_{\mathbf{x}}$ qui serait due à la recombinaison de l'exciton libre, puis aux environs de $2,795 \mathrm{eV}$ une série de raies très intense responsable de la couleur violette de l'échantillon. Ces raies difficilement séparables ont des amplitudes relatives qui varient considérablement

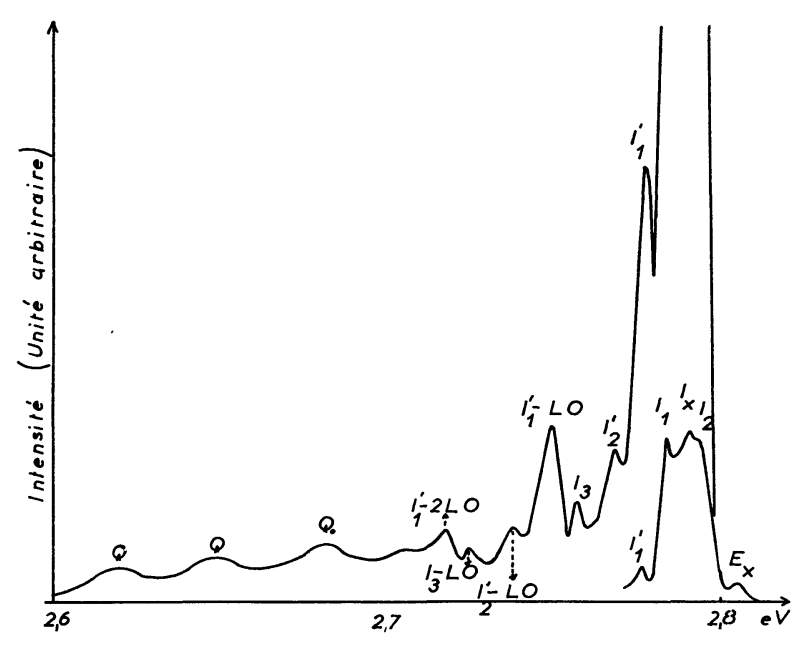

FIg. 2. - Répartition spectrale de la lumière émise par les cristaux du type I excités par des électrons de $15 \mathrm{kV}$ à la température de $13 \mathrm{~K}$.

suivant le point d'impact du faisceau électronique sur le cristal. Nous avons pu cependant identifier sur certains clichés un triplet dont une composante $\mathbf{I}_{2}$ à $2,795 \mathrm{eV}$ serait due à des transitions d'excitons liés à un donneur neutre, une composante $I_{x}$ à $2,789 \mathrm{eV}$ et une composante $I_{1}$ à $2,783 \mathrm{eV}$ due à la transition d'excitons liés à des accepteurs neutres [9], [10].

$\mathrm{La}$ raie $\mathrm{I}_{1}^{\prime}$ environ 5 fois moins intense que les raies du triplet, placée vers $2,778 \mathrm{eV}$ pourrait être due à une réplique avec émission de phonon de la raie $\mathrm{E}_{\mathrm{x}}$ et présente elle-même 2 répliques avec émission de phonons LO. Au pied de cette raie se trouvent 1 et parfois 2 pics du côté des basses énergies. Nous pensons que le pic noté $I_{2}^{\prime}$ serait dû à $I_{2}-$ TO. Les attributions des autres raies sont indiquées sur la figure 2. (Les fréquences LO et TO ont été mesurées sur nos échantillons grâce à l'obligeance de $\mathbf{M}$. Beserman.)

L'émission spectrale se termine par 3 bandes larges très peu intenses (nettement moins intenses que celles observées par Dean et Merz et surtout Reynolds). Elles seraient dues à des transitions de donneuraccepteur associées aux environs de 2,682 eV; $2,648 \mathrm{eV}$; $2,620 \mathrm{eV}$ et se rapprochant des bandes $\mathrm{Q}_{0}$ présentées par Dean et Merz.

Les émissions spectrales des cristaux du type II, de couleur bleue, sont catractérisées par l'existence d'une émission intense due à des pièges peu profonds, ou émission de bord de bande et d'une série de raies 
au voisinage de la limite d'absorption. La répartition spectrale et les rapports d'intensité des différentes raies émises sont très voisins de ceux trouvés dans la littérature [9], [10], [11]. Nous avons retrouvé ainsi vers $2,802 \mathrm{eV}$ la raie due à l'exciton libre, et les raies $\mathrm{I}_{2}, \mathrm{I}_{\mathrm{x}}$ et $I_{1}$ situées respectivement à $2,796 \mathrm{eV} ; 2,790 \mathrm{eV}$; $2,785 \mathrm{eV}$. Ces raies sont mieux définies que les raies des cristaux du type I et leurs intensités sont différentes: $I_{2}$ est moins intense, $I_{x}$ est faible et $I_{1}$ est plus intense. La raie $I_{1}$ est suivie d'une forte réplique avec émission de phonon LO.

Les attributions des autres raies du spectre, la position des bandes dues à des transitions de paires donneur-accepteur associées ainsi que la structure haute énergie de la première bande, sont indiquées sur la figure 3 .

3. Conclusion. - Le dispositif expérimental que nous venons de décrire nous a permis d'améliorer l'étude par cathodoluminescence du séléniure de zinc, en limitant les conséquences des fluctuations des paramètres d'excitation et des températures dans l'obtention des spectres d'émission point par point de nos échantillons.

L'interprétation que nous avons proposée est celle que l'on peut déduire des travaux de Thomas et Hopfield, Dean et Merz, et Langer qui est la plus couramment admise. Notre dispositif doit permettre une étude plus précise de l'influence des paramètres d'excitation sur les différents échantillons afin d'améliorer certaines interprétations.
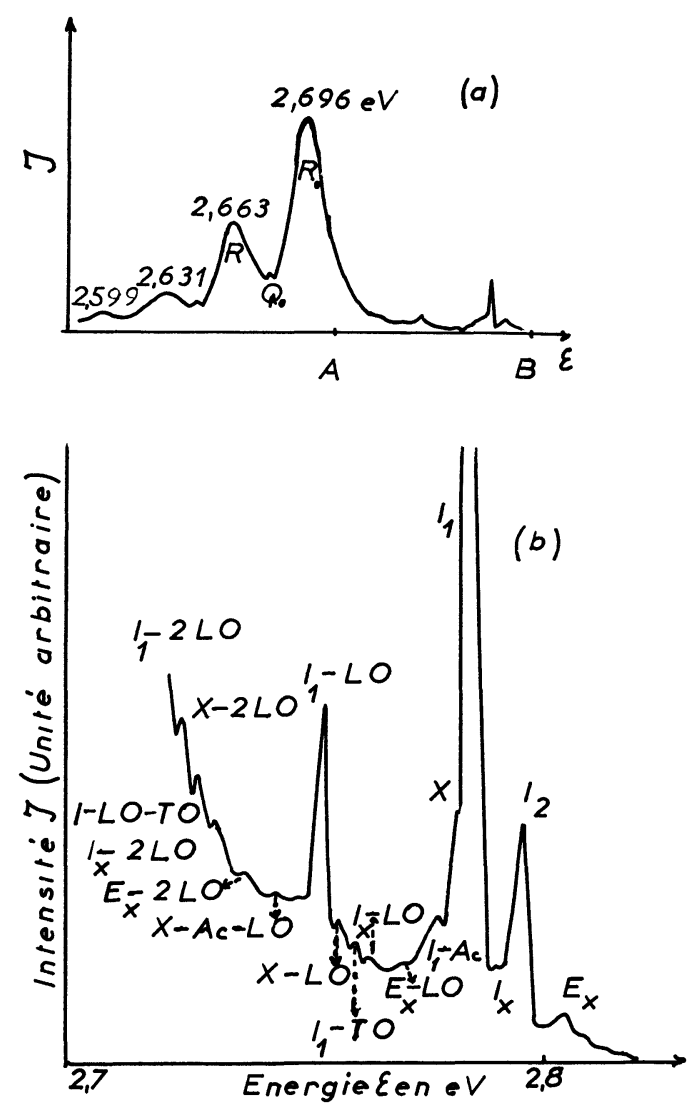

Fig. 3. - Répartition spectrale de la lumière émise par les cristaux du type II, excités par des électrons de $15 \mathrm{kV}$, à la température de 13 K. a) Spectre d'ensemble. b) Agrandissement de la partie AB.

\section{Bibliographie}

[1] BALKANSKI, M., GANs, F., Luminescence of organic and inorganic materials (édité par H. P. Kallmann et G. M. Spruch, Wiley) 1962318 .

[2] Benoit-A-la-Guillaume, C., Bonnot, A. et Debever, J., Phys. Rev. Lett. 24 (1970) 1235.

[3] Hrtier, G. et Gans, F., J. Physique 28 (1967) C 3-69.

[4] Reynolds, D. G., Litton, C. W. et Collins, T. C., Phys. Stat. Sol. 9 (1965) 643.

[5] Thomas, D. G. et Hopfied, J. J., Phys. Rev. 128 (1962) 2135.

[6] Sobolev, V. V., Optics and Spectroscopy 16, 40 (1964) 368.
[7] Guillard, J. M., Thèse de Doctorat d'Université Paris, juin 1967.

[8] Reynolds, D. C., Pedrotti, L. S. et Larson, O. W., J. Appl. Phys. 32 (1969) 2250.

[9] KoH ERA et LANGER, D. W., J. of Luminescence 1-2 (1970) 514.

[10] Dean, P. J. et Merz, J. L., Phys. Rev. 178 (1969) 1310.

[11] Fujwara, S., Hattori, K. et Fukai, M., Phys. Lett. 31A (1970) 258. 